\title{
Entre discours et pratiques : esquisse d'un état des lieux de la société de l'information
}

Groupe de recherche Technologies et Modernité, Gérald Berthoud, Daniela Cerqui, Farinaz Fassa et Frédéric Ischy

\section{OpenEdition}

\section{Journals}

Édition électronique

URL : http://journals.openedition.org/ress/600

DOI : 10.4000/ress.600

ISSN : 1663-4446

Éditeur

Librairie Droz

Édition imprimée

Date de publication : 1 juin 2002

Pagination : 5-19

ISBN : 2-600-00693-1

ISSN : 0048-8046

Référence électronique

Groupe de recherche Technologies et Modernité, Gérald Berthoud, Daniela Cerqui, Farinaz Fassa et Frédéric Ischy, «Entre discours et pratiques : esquisse d'un état des lieux de la société de

l'information », Revue européenne des sciences sociales [En ligne], XL-123 | 2002, mis en ligne le 01 décembre 2009, consulté le 22 avril 2019. URL : http://journals.openedition.org/ress/600 ; DOI :

$10.4000 /$ ress.600 


\section{ENTRE DISCOURS ET PRATIQUES : ESQUISSE D'UN ÉTAT DES LIEUX DE LA SOCIÉTÉ DE L'INFORMATION}

Plus que l'expression elle-même de société de l'information (SI), les idées associées à cette formule ne datent pas d'hier (Simioni, 2000). Ainsi, figure importante du renouveau actuel de la constellation sémantique dans laquelle gravite la notion de SI, le sociologue américain Daniel Bell affirmait il y a près de trente ans déjà que «si la société industrielle est une société de production des biens, la société postindustrielle est une société de l'information» (Bell, 1976: 402). Le passage à une économie de services ainsi que la centralité du «savoir théorique » et des nouvelles «technologies de l'intellect» dans l'innovation tant économique que sociale caractériseraient alors selon lui cette nouvelle forme de société ${ }^{1}$. Il apparaît toutefois possible et légitime de remonter plus avant encore dans le temps. A ce titre, on peut indiquer la réflexion et les développements technoscientifiques initiés dès les années 1940. Les références obligées sont dès lors Claude Shannon (1949) dont la théorie mathématique de la communication permet de calculer la quantité d'informations à partir d'une mesure élémentaire (le bit), et Norbert Wiener (1952) dont la cybernétique inaugure la perception du réel comme flux d'informations susceptibles, le cas échéant, de traitement automatique. Peut-être même conviendrait-il de remonter jusqu'aux sources du mouvement moderne de rationalisation (Mattelart, 2001) et de prendre en compte le fait que la «mathématisation du monde [consistant] à introduire la quantité et l'ordre, donc le nombre, comme principes de description d'un domaine et comme outils d'une action de transformation de ce domaine» remonte au XVIIème siècle avec l'application de principes constituant «le fondement incontesté de la démarche scientifico-technique dans les sociétés occidentales » (Scardigli, 2001 : 137-138).

Idée ancienne, la notion de SI - certes parée des oripeaux de la «grande nouveauté »- ne jouit pas moins de nos jours d'un retentissement considérable. Véritable leitmotiv des décideurs des milieux tant économique, politique, scientifique que médiatique, le terme de SI et les thèmes afférents s'insinuent partout, en dépit des fortunes diverses que vit la saga d'Internet et de la dite «nouvelle économie » qui lui est liée. Ce succès d'estime s'accompagne pourtant de trop peu d'études d'envergure sur le sens profond de la notion de SI et sur les implications théoriques et pratiques des représentations qui la sous-tendent. Signalons à ce propos les récents examens historiques d'Armand Mattelart (2000, 2001), ceux, plus analytiques, d'Alistair Duff (2000) ainsi que nos propres travaux (Berthoud et al.,

Bell va actualiser ses idées dans l'avant-propos qu'il donne à l'édition de 1999 de The Coming of Post-Industrial Society. New York: Basic Books. 
2000, 2002). Cette investigation critique reste cependant encore largement à faire. C'est dans le but d'ébaucher des perspectives en vue de la poursuite de cette vaste tâche d'enquête raisonnée et argumentée que nous avons réuni dans ce numéro un ensemble de textes, susceptibles de dresser un état des lieux de la réflexion en la matière.

A la question de savoir ce qu'est la SI, nous nous trouvons confrontés à une pluralité de réponses, et il apparaît en conséquence que la SI s'insère dans une sorte de nébuleuse sémantique: tour à tour qualifiée de fiction, d'idéologie, d'utopie ou de mythe voire de culte, notamment par les textes présentés ici, elle est pour d'autres une réalité tangible indéniable. Ainsi, même si l'on en reste à la caractérisation de la SI en tant que discours, un certain «brouillard» règne et mériterait d'être dissipé. Patrice Flichy (2001a: 12-16) donne au sujet de ce dernier point une piste intéressante. En s'appuyant en particulier sur la réflexion de Paul Ricœur (1997) concernant la tension entre, d'une part, l'idéologie qui légitime le pouvoir en place et préserve l'identité du groupe social et, d'autre part, l'utopie qui constitue une alternative au pouvoir en place et explore les possibles, Flichy esquisse un modèle où le discours sur les technologies de l'information et de la communication (TIC) et sur la SI passerait par des phases distinctes. La première de ces phases serait celle, chaotique, de la gestation de l'innovation où le discours prendrait la forme d'une «utopie de rupture». Puis suivrait une phase de concrétisation à l'issue de laquelle la réflexion utopique soit s'incarnerait dans un projet expérimental, une «utopie-projet», soit échouerait ou renoncerait à se confronter à la réalité (technique et sociale) et se perdrait ainsi dans une «utopie-fantasmagorie». Dans la phase de tests, les expériences réussies seraient transformées en mythes, c'est-à-dire en récits dont la signification dépasse les simples anecdotes qui en sont à la base. Les TIC et leurs réalisations locales finiraient par être présentées comme la technique de base d'un nouveau fonctionnement social, le mythe transformant alors l'utopie en «idéologie-masque», "idéologie légitimante » et/ou «idéologie-mobilisation » (Flichy, 2001b).

Quoi qu'il en soit, pur discours ou réalité objectivable indépendante des observateurs, le statut ontologique de la SI est sujet à controverses. En tous les cas, cette «nouvelle forme sociale» échappe à toute définition univoque. Schématiquement, il est possible de situer toutes les définitions repérables sur un axe qui va de la rêverie plus ou moins fantaisiste (voire parfois de la divagation) à la prétention que la SI constitue pleinement un fait «brut», observable comme une chose. La rigueur scientifique, mais aussi peut-être l'honnêteté politique, devrait imposer aux acteurs prééminents de la SI de se positionner le plus clairement possible sur ce continuum. Et l'on sent bien que la réalité, faite de nuances, ne pourra alors jamais strictement se réduire à l'un ou l'autre des pôles de l'axe envisagé. Que la SI relève plutôt du discours ou plutôt de phénomènes et comportements extérieurs à l'observateur, il n'en reste pas moins que, à suivre Alistair Duff (2000), les Information Society Studies représentent un domaine d'étude concret et légitime. Nous ajouterons pour notre part que, afin de mettre un terme provisoire au débat sur la nature véritable de la SI et afin de permettre aux sciences sociales d'engager et de poursuivre valablement les «études SI», la notion de SI a tout lieu d'être caractérisée et utilisée heuristiquement comme un idéal-type weberien (cf. Ischy).

Force est de constater que les textes rassemblés ici sont le signe évident que les chercheurs en sciences sociales francophones ne considèrent pas la SI comme 
allant de soi et s'accordent à la questionner. En effet, l'état des lieux qu'ils nous dépeignent porte bien plus sur de la rhétorique que sur une réalité considérée comme objective. Tous ces textes ont alors en commun une volonté de déconstruire le discours sur la SI, les nouvelles technologies et, plus généralement, les techniques et leur rapport au social. Ils sont autant de tentatives d'englober à la fois l'aspect matériel et l'aspect idéel qui lui est intimement lié, même si la plupart insistent avant tout sur l'aspect discursif. Ils ouvrent en cela une piste pour réfléchir aux types d'interactions qui régissent les rapports entre discours et pratiques. Quelques remarques générales peuvent être émises à ce propos. Il convient premièrement d'insister sur l'imbrication consubstantielle des récits et des pratiques. Il est dès lors inadéquat, à notre avis, de ne considérer qu'un récit dominant tout en renonçant à s'intéresser aux technologies elles-mêmes, comme si les deux pouvaient être déconnectés. Dans cette perspective, l'étude des usages des techniques, des TIC en l'occurrence, s'avère cruciale et, à nouveau, reste encore largement à faire. Ensuite, le fait qu'il y ait d'une part une réalité de type technologique et d'autre part toute sorte de discours qui tendent en partie à s'autonomiser de cette réalité donne lieu à des représentations fragmentaires. Il devient alors nécessaire de contextualiser les différents discours et de savoir à quels types d'acteurs et d'actions concrètes ils renvoient. Il est ainsi important de saisir qui est le locuteur pour comprendre le sens réel du discours. Par exemple, Al Gore, durant la campagne présidentielle de 1992, parle d'autoroutes de l'information en hommage à son père qui «comme sénateur, joua un rôle important dans le lancement du programme d'autoroutes inter-Etats dans les années cinquante» (Flichy, 2001a: 27). Ce faisant, il inscrit le projet à la fois dans l'histoire des réseaux de circulation et de l'intervention de l'Etat en la matière et, à la fois, dans son histoire propre. Finalement, il importe d'évaluer les écarts et de comprendre les raisons au fondement des hiatus non seulement entre pratiques et récits mais aussi entre ces derniers eux-mêmes. Toujours à en croire Flichy (2001a: 114), les discours qui aident habituellement à la diffusion des techniques n'ont souvent «plus rien à voir avec les débats initiaux des concepteurs », au contraire du cas d'Internet dont «l'imaginaire proposé aux utilisateurs s'inspire largement des utopies des concepteurs mais [...] a néanmoins subi un certain nombre de transformations ». A l'appui de cette thèse viennent les résultats de nos propres travaux où l'on constate que les visions des praticiens de la SI, qu'ils se situent aux niveaux technoscientifique, économique et/ou politique, tout en se référant aux fantasmagories du début s'en écartent beaucoup à travers une perception très immédiate et pragmatique des enjeux liés aux TIC (Berthoud et al., 2002). Car, après tout, à côté d'expérimentations sociotechniques réussies, il en est qui rencontrent moins de succès, voire qui échouent.

\section{UNE PERPÉTUELLE «NOUVEAUTÉ»}

Malgré un foisonnement disparate, les vulgates consacrées à la SI s'unifient dans leur emploi commun d'un langage révolutionnaire. Nouveauté véritable, c'est-à-dire en rupture radicale avec l'ordre ancien des choses, la SI marquerait alors notre entrée dans l' «Age de l'information», l' «Ere digitale» ou la «Civilisation du numérique». Cette rhétorique, articulée plus ou moins consciemment et 
avec plus ou moins de conviction, traduit ainsi une conception «développementaliste » de l'histoire des sociétés (cf. Ischy) où des types sociaux sont mis en avant et considérés comme autant d'étapes successives d'un même développement. Cette inscription dans une périodisation sur un temps long et ce qui apparaît comme une «théorie des stades » peut plus ou moins implicitement faire le lien, de cas en cas, avec certaines formes d'évolutionnisme et de vues téléologiques proposant une hiérarchisation des sociétés à partir de valeurs prioritairement occidentales.

Mais comment rend-on compte généralement de cette nouveauté, du passage à cette nouvelle société que symboliserait la SI? L'aspect «inédit» est principalement défini selon des critères économiques et techniques, dont différentes formes se sont déjà succédées ces dernières années, de la «révolution microélectronique » à la «cyber-révolution» (Robins et Webster, 1999), avec actuellement la convergence des industries de l'audiovisuel, de l'informatique et des télécommunications permise par la numérisation ainsi que le bond opéré par les échanges économiques qui se nouent autour du stockage, du traitement et de la diffusion de l'information. Peut-on valablement qualifier une société prioritairement à partir de sa dimension technique (et/ou économique)? Il y a tout lieu de penser que nous sommes en présence ici d'un réductionnisme qui fait «prendre la partie pour le tout» (cf. Sfez).

La technique est alors conçue comme le facteur essentiel du changement social et comme la cause au sens plein du développement économique et, partant, de l'évolution sociale. Plutôt que d'envisager une telle linéarité et un tel déterminisme du techno-économique sur les autres sphères du social, une approche pertinente consiste à interroger les éléments qui «accompagnent» les changements considérés (cf. Miège). Goody (1979, 1986), bien que souvent accusé de déterminisme, propose une lecture plus nuancée de ces relations. Plus largement, il convient à notre avis de mettre en lumière à quel point les techniques sont elles-mêmes le résultat d'un contexte (économique, social, culturel, etc.) particulier et apparaissent dès lors comme des constructions sociales (voir par exemple Miquel et Ménard, 1988). Dans cette optique, la métaphore de l'impact (les technologies, les TIC en l'occurrence, sont là et on en étudie les effets - positifs et négatifs - sur/dans la société comme on analyserait les conséquences de la chute d'un météorite sur la croûte terrestre) doit être écartée autant que possible. Même si la réflexion scientifique consiste justement à faire la part des choses, cette lecture en termes d'impact, sans cesse mise en œuvre dans le discours actuel sur la SI, reste pourtant le plus souvent à bannir tant, dans la réalité, causes et effets, « déterminants » physiques, matériels, cognitifs, etc. (toujours partiaux) et «déterminants» sociaux (de même toujours partiaux) se mêlent. Dans cette même perspective, les technologies, conçues désormais comme des «dispositifs» sociotechniques, ne s'accommodent alors guère plus du recours répété à la prétendue neutralité (structurelle, éthique...) de la technique. D'ailleurs, de façon paradoxale mais révélatrice, cette «neutralité» se trouve continuellement mise en avant dans la rhétorique relative à la SI ( «les TIC ne sont ni bonnes ni mauvaises, et la SI sera ce que nous en ferons») tout en étant couplée à l'annonce de l'inéluctabilité de l'avènement de la SI («adaptez-vous ou disparaissez!»).

La SI, une nouvelle société? Nous avons mentionné en introduction le caractère relativement ancien, si ce n'est de l'expression elle-même de SI, du moins des idées associées à cette formule. Nous constatons que souscrire à la qualification 
de la SI comme forme sociale radicalement nouvelle traduit une conception très problématique de l'histoire des sociétés et reflète une appréhension passablement inadéquate du «fait» technique. Nous nous apercevons par ailleurs que, depuis le XIXème siècle, toute innovation technique majeure engendre des vues prophétiques (voir, par exemple, Marvin, 1988). Nous pourrions ainsi rechercher de solides traces de la «nouvelle» société dans les discours d'accompagnement, qui se développèrent en relation avec des technologies de la communication comme le télégraphe, il y a plus de cent cinquante ans. A ce titre, la philosophie saintsimonienne et sa postérité actuelle forment une illustration exemplaire (cf. Musso). A partir de ce que le passé nous enseigne, nous pouvons donc avancer très sérieusement que «Today's future is yesterday's future as well» (Carey et Quirk, 1989: 182). La SI apparaît dès lors bel et bien comme un des derniers avatars de la continuelle prévision d'une forme de «vivre ensemble» profondément inédite.

\section{DES ÉTIQUETTES DIFFÉRENTES}

Le passage vers «la société de l'information» est présenté comme une entrée dans un temps radicalement neuf, comme l'imminente transition vers une société qui permet une relation des uns aux autres pacifiée et dénuée des conflits qui ont agité le temps industriel. La perception technocentrée du social qui a vu le jour sous la plume des futurologues vers 1970 a trouvé un nouvel écho sous celle des politiques ou des financiers; elle s'est aussi parée d'autres attributs. Renaissante, elle n'a pas renié ses précurseurs, mais elle y a ajouté d'autres dimensions qui tiennent toutes à l'évolution que la technique a vécue durant ces trente dernières années. L'information des cybernéticiens a été confondue avec l'information, «produit que l'on peut trouver dans un dossier, un livre, une bibliothèque ou dans une quelconque 'banque de données'» (Gouldner, 1989: 17), et partant elle a été associée au savoir. Cette équivalence est visible dans la façon dont les textes qui traitent de la société de l'information en tant que « société à faire advenir » confondent ces deux notions (cf. Fassa) qui sont pourtant bien éloignées puisque, selon Gouldner (1989: 17), le savoir, au-delà d'une somme d'informations dépersonnalisées est aussi une conscience qui implique «l'effort de sujets totaux et incarnés». Les expressions qui associent savoir ou connaissance à information sont devenues légions et «la société de l'information» et «la société de la communication» ont trouvé un nouveau double, «la société du savoir ou de la connaissance». Ce dernier avatar s'est surajouté à une nébuleuse confuse qui comprenait déjà la communication et ses moyens techniques, les connexions qui établissent la relation grâce aux réseaux.

Apparentées quant à leurs objectifs et à leurs modalités de mise en récit de la technique (cf. Sfez), ces diverses expressions offrent cependant des éclairages différents sur ce que la technique fait advenir ou sur ce qu'elle accompagne.

Il convient d'au moins mentionner brièvement ce qui s'inscrit dans ces différentes métaphores car la façon de déconstruire ces expressions, « mots-valises », nous interroge sur le sens d'un éventuel désenchantement (cf. Breton) face à la «société de l'information». Parler de «la société de l'information» ou mettre l'accent sur «la société de communication» ne renvoie pas tout à fait au même 
univers de sens. Si Internet tient surtout à la communication et a marqué la dernière décennie d'une façon telle que «la toile» soit devenue tout à la fois symbole et manifestation d'un changement appelé celui de «la société de l'information», ce réseau de circulation des informations ne peut être associé à l'ensemble de ce qui se trame dans cette transition: le désamour qui atteindrait Internet ne signifierait pas alors que «la société de l'information» soit elle aussi une notion en crise. Il se pourrait même que l'usage d'Internet soit devenu suffisamment évident pour tous pour que son abord soit avant tout instrumental. Ne plus faire l'objet de débats publics, ne plus être une technologie mobilisatrice ou une fiction fondatrice ne signifierait alors pas que «le thème de la société de l'information tend à voir son influence décroître » (cf. Breton) mais bien plutôt qu'il s'agirait d'une référence stabilisée.

Les «Technologies de l'Information et de la Communication» mêlent en elles deux aspects qui nous paraissent devoir être distingués pour préciser les articulations sémantiques qui, sous une apparente unicité rhétorique (la SI), renvoient à des imaginaires qui construisent tout autant l'image d'un humain émancipé et prêt à devenir un sujet que celle d'un être hybride perdu dans un corps et un esprit numérisés.

L'information, au-delà de sa compréhension cybernéticienne, appelle très rapidement le savoir ou la connaissance et l'équivalence entre ces notions se donne comme évidente puisque toutes trois sont souvent considérées comme des forces productives. Nous en voulons pour exemple le passage de l'économie de l'information à celle du savoir et les tentatives faites par le Groupe des Experts de Haut Niveau (1997) pour délimiter le champ de l'une et de l'autre et tracer une frontière bien peu marquée puisqu'en perpétuelle mouvance. L'information n'appelle pas aussi nettement la communication et c'est en rapport avec les instruments techniques que ces deux adjectifs ont été accolés d'une manière telle qu'ils paraissent parfois indissociables. Mais à la communication s'apparentent les relations que les personnes peuvent entretenir et c'est sur ce fondement que se construisent les utopies les plus résolument anti-hiérarchiques et égalitaires. Ce terreau communicationnel et réticulaire sert cependant aussi bien à l'éclosion des récits du capitalisme libéral qui privilégient les individus, noyaux indistincts mais reliés temporairement par des projets que les connexions permettent de mener à bien qu'à des utopies d'inspiration libertaire et/ou égalitaire.

La «société de l'information» et la «société de la communication» nous semblent ainsi renvoyer à deux récits bien souvent confondus de par l'importance prépondérante que les chercheurs, comme les politiques, ont accordée durant cette dernière décennie à Internet, outil de circulation des informations et de communication entre usagers.

\section{SOCIÉTÉ DE L'INFORMATION ET SOCIÉTÉ DU SAVOIR}

L'équation «plus d'information = plus de savoir» devient le centre névralgique de ces discours et la distinction entre ces deux concepts n'est que rarement interrogée. La confusion qui règne autour de la définition de la SI (cf. Simioni et Ischy) rejaillit sur tout ce qui lui appartient et l'outil numérique, moyen d'acqué- 
rir des informations et de constituer un savoir, devient fin en soi. Ce glissement s'effectue d'autant plus facilement que des chercheurs lui prêtent, en tant que technologie intellectuelle, des qualités qui l'inscrivent dans une réflexion exclusivement informationnelle: lieux de stockage des informations et bases de données servant à constituer des savoirs, les ordinateurs deviennent des nœuds sur les chemins de la connaissance et sont assimilés, dès lors qu'ils se trouvent en réseau, aux neurones cérébraux (cf. Berthoud). La proximité des termes qui entouraient l'objet technologique lui-même crée une équivalence qui dépasse largement la description des usages diversifiés que l'on peut en faire. L'information et le savoir sont, dans cette conception, considérés comme des synonymes presque parfaits et cette équation sert aujourd'hui de base aux discours sur le digital divide, qui confond accès au contenant et accès au contenu.

Les nombreuses dimensions relatives à l'acquisition du savoir, liées, par exemple, à la relation entre l'éducateur et son élève, à l'implication des individus dans leur entièreté dans le processus d'acquisition des connaissances, sont ainsi évacuées. Nos différentes enquêtes empiriques - portant sur des domaines tels que l'école à l'heure de la société de l'information, les représentations de la société de l'information chez des dirigeants du monde politique, économique ou scientifique ou encore l'image que se font toute une série d'ingénieurs de ce qu'est et doit être un être humain - montrent en effet toutes ce glissement qui tend à faire du savoir quelque chose de directement applicable et utilisable, quelque chose qui peut être divisé, échangé et monétarisé, bref, un bien qui peut être productif. La connaissance qui permet à chacun de participer à un ensemble social et d'y tenir sa place en tant que citoyen est ainsi transformée en instrument qui agit sur les individus en les contraignant au lieu d'agir dans les individus en leur permettant de se construire (Fassa, 2002).

«Avec l'hégémonie de l'informatique, c'est une certaine logique qui s'impose, et donc un ensemble de prescriptions portant sur les énoncés acceptés comme 'savoir'. On peut dès lors s'attendre à une forte mise en extériorité du savoir par rapport au 'sachant'... L'ancien principe que l'acquisition du savoir est indissociable de la formation (Bildung) de l'esprit et même de la personne, tombe et tombera davantage en désuétude. Ce rapport des fournisseurs et des usagers de la connaissance avec celle-ci tend et tendra à revêtir la forme que les producteurs et les consommateurs de marchandises ont avec ces dernières, c'est-à-dire la forme valeur. Le savoir est et sera produit pour être vendu, et il est et sera consommé pour être valorisé dans une nouvelle production: dans les deux cas, pour être échangé. Il cesse d'être à lui-même sa propre fin, il perd sa 'valeur d'usage'. On sait qu'il est devenu dans les dernières décennies la principale force de production» disait il y a vingt ans déjà Lyotard (1979: 13-14).

N'expliquait-il pas alors les fondements de l'opération qui a consisté à transformer en équivalence « information » et «savoir», à présenter comme des notions tenant l'une pour l'autre «société de l'information» et «société du savoir»?

Ce qui avait été envisagé comme une possibilité, un droit à conquérir - celui d'une éducation de qualité pour tous - tend aujourd'hui à devenir une contrainte, celle de se former continuellement et pour cela de se connecter aux réseaux informatiques, bases «d'un monde meilleur et plus horizontal», voire dans l'esprit de certains $d$ ' «une intelligence collective». La force que le savoir peut donner au 
sujet a ainsi été neutralisée et le sens qui était donné à l'éducation et à la formation modifié (cf. Fassa).

Les fortes incitations à la formation permanente ayant surtout pour objectif de pouvoir répondre aux demandes de flexibilité que formulent les entreprises, la formation continue est devenue une fin en soi et l'objectif des écoles est souvent, aujourd'hui, de créer des dispositions à apprendre chez des individus, plutôt que d'inculquer des savoirs constitutifs de sujets autonomes. S'il s'agit de former de futurs travailleurs, censés pouvoir traiter au plus vite des données relatives au fonctionnement d'une entreprise, il semble en effet que l'on puisse se contenter pour l'éducation de la majorité des jeunes de définitions réductrices ou réduites du savoir. Par contre, s'il s'agit d'éduquer des citoyens, censés pouvoir se construire une opinion et la défendre dans un espace public démocratique, il faut aller plus loin et accepter de voir qu'au-delà de l'accès à l'information, la question de la connaissance exige de réfléchir plus largement au type de société et d'homme que ces notions proposent implicitement.

Appréhender la construction des connaissances, même dans une société fortement marquée par l'essor des technologies numériques et par leur omniprésence, dans des termes qui ne s'articulent qu'autour de la question de l'accès versus du non-accès à l'information est insuffisant et trompeur. S'il nous apparaît qu'il est possible d'évoquer un digital divide ou une «fracture numérique» dans le sens où il existe un accès différencié aux technologies de l'information et de la communication, et ceci aussi bien pour des raison liées au coût financier élevé de ces technologies que pour des motifs géographiques ou de positionnement dans l'espace social, il ne nous semble cependant pas que cet accès différencié aux technologies elles-mêmes puisse être unilatéralement étendu à un accès différencié à l'information, et encore moins à un accès différencié au savoir. Une telle perspective suggère que les NTIC sont comprises comme des instruments permettant naturellement un surplus de connaissances, puisqu'elles mettent à disposition sur les réseaux des informations pouvant servir de fondements à la construction de savoirs.

Il faut, selon nous, aller au-delà de cette question, dont on ne peut par ailleurs négliger les nombreux défis, et aborder les enjeux de définition qui sont fondamentaux puisque selon la définition que l'on propose du savoir - et de sa relation avec l'information -, les politiques de formation ou d'éducation seront différentes.

On ne peut se contenter d'une vision du savoir qui en fasse une accumulation d'informations ou de données «cueillies » sur le réseau des réseaux, comme on ne peut se contenter d'aborder la communication à travers ses réseaux. Dès lors, avant de se demander s'il existe un digital divide faisant que seules certaines personnes ont la possibilité d'accéder à un nouvel état de connaissance, à un nouvel état de relation, encore faudrait-il savoir si, effectivement, les «nouvelles » technologies en augmentant l'accès à l'information et en facilitant les échanges sur les réseaux ont le pouvoir d'augmenter l'accès au savoir et la communication entre les sujets? 


\section{SOCIÉTÉ DE LA COMMUNICATION ET SOCIÉTÉ EN RÉSEAUX}

Le fait de mettre exclusivement l'accent sur les autoroutes de l'information, et en particulier sur Internet, a pour avantage de mettre en évidence la promotion d'un nouveau mode de vivre "en réseaux», qui correspond à un mouvement de virtualisation du réel et qui se veut en rupture avec toute inscription territoriale concrète. Or, la notion même de réseau, fortement polysémique (elle peut désigner des flux, des lieux, des infrastructures ou des personnes, voir Bakis, 1993), est initialement indissociable de l'espace matériel, puisqu'elle s'est appliquée à l'aménagement territorial (réseau d'eau ou réseau électrique par exemple) bien avant de désigner la virtualisation du social.

Mais de nos jours, bien plus qu'un simple principe d'organisation spatiale, le réseau est devenu le symbole des structures non hiérarchiques, de la communication et de l'égalité. Le poids est ici sur le lien, la relation, ce qui permet d'échapper à un ancrage temporel et spatial parfois pesant, puisqu'il nous rappelle notre appartenance et notre existence en tant que membres de communautés réelles et territoriales. En appeler à la relation, c'est en référer à l'instauration d'un «monde où les êtres ont donc pour préoccupation naturelle le désir de se connecter aux autres, d'entrer en relation, de faire des liens, de ne pas demeurer isolés» (Boltanski et Chiapello, 1999: 168). Les individus, hors de leurs groupes d'appartenance traditionnels, sont présentés par ce récit communicationnel comme «libres» de recréer des liens avec d'autres individus tout aussi «libres ». Chacun serait en droit de choisir de communiquer avec un autre, élu par lui; même éloignés dans l'espace, les correspondants resteraient proches dans les préoccupations ou dans les projets. Les communautés traditionnelles et leurs pesanteurs seraient ainsi vaincues, les individus triompheraient et s'épanouiraient dans des univers exempts de la violence que l'autre inflige par sa radicale différence. Selon les zélateurs de la «société de la communication», l'«outil» numérique serait à la source d'une horizontalisation des rapports sociaux, il contribuerait à la reconnaissance de tous puisqu'il favoriserait un éclatement des centres de pouvoir par la distribution non hiérarchisée des «paroles » qui, à travers l'espace de la «toile » pourraient se rencontrer et se rejoindre.

Cette vision séduisante rallie de manière surprenante les tenants du discours dominant, managers d'un nouveau capitalisme pour qui un tel déracinement signifie la flexibilité voulue, et les défenseurs d'une certaine vision libertaire. Cette rencontre autour du thème de l'individu et de sa liberté (que l'on peut aussi comprendre comme une désappartenance) intervient en effet tant dans les discours que certaines franges modernistes de la gestion libérale de l'Etat, de l'économie et de la déréglementation nous infligent que dans certaines des réflexions des mouvements sociaux. Pour ces derniers, les réseaux paraissent des lieux de paroles et d'échanges qui échappent au contrôle des pouvoirs institués.

«Le réseau porte toujours en lui un imaginaire de la transition, entre la libération d'un système pyramidal et hiérarchique dont l'Etat est l'archétype, et la promesse d'un système futur, celui de l'association universelle, annonciateur d'un nouveau type de relations égalitaires » (Musso, 1998: 2329). Intrinsèquement porteur d'un futur libérateur, il renvoie au mythe développé de nos jours par la société de l'information, dans laquelle les réseaux devraient ouvrir une ère nouvelle. 
Dans cette optique plus qu'optimiste, les réseaux apparaissent comme des «machines relationnelles qui offrent à leur membres un cadre pour leurs relations et invitent à l'élaboration de projets communs » (Bressand et Distler, 1995: 191). Pour les mouvements sociaux, ils représentent l'occasion de faire connaître et de diffuser des informations, d'utiliser les «outils» de la «globalisation pour lutter contre la mondialisation» ou ceux de «la technologie masculine pour lutter contre le patriarcat». Permettant tout à la fois de coordonner des actions politiques en assurant une communication plus rapide et plus fréquente entre les militants et les organisations partenaires et d'informer sur les objectifs de certaines actions, les TIC semblent receler des potentialités importantes, y compris de manifester par sa domination technique le fait que l'on n'appartienne pas à un monde révolu, condamné à disparaître ou à être dominé.

C'est bien évidemment autour de ces questions identitaires que la métaphore du réseau se déploie dans toutes ses composantes rhétoriques et que se dessine la confusion entre réappropriation des techniques et horizon libéré. Les interrogations identitaires sont aussi celles qui font glisser le «réseau» hors de la question exclusive de la communication - et de la fiction de la transparence du lien immédiat qui a supprimé toute autre médiation - pour atteindre celle de l'information.

Le cas des zapatistes est bien connu mais il mérite d'être mentionné; les populations autochtones, en faisant usage d'Internet, n'ont pas seulement trouvé un moyen pour diffuser des informations sur leurs situations de dominés et agréger des volontés militantes et de soutien, elles ont aussi souligné que leur combat n'était en rien passéiste et comme tel voué à être perdu. Dominant les techniques «nouvelles » de la communication et de l'information et le clamant à la face de tous par leur présence sur la «toile», cette organisation a revendiqué un statut dans la modernité pour les populations autochtones et affirmé que leurs modes d'existence n'étaient en rien incompatibles avec le monde du XXIème siècle.

Le réseau des réseaux, en désenclavant une protestation identitaire et anticapitaliste, a servi à poser un jalon qui tout en même temps dit la volonté de l'ancrage territorial, culturel et communautaire mais aussi la possibilité pour celui-ci de subsister dans un temps de mutation qui privilégie le global, le mondial et le nouveau. S'agit-il pour autant de ce qu'Isabelle Pailliart, dans la ligne des experts de la Commission européenne, nomme «reconfiguration des pouvoirs» ou d'une appropriation particulièrement réussie des techniques de la communication et de la diffusion des informations par des protestataires? S'agit-il en d'autres termes d'un usage conscient de l' " outil » comme prolongement des finalités humaines ou de l'oubli que la transparence a son revers et qu'elle implique la visibilité de tous par tous et peut dès lors aussi être synonyme de contrôle?

La réponse ne saurait être simple et unique comme nous le rappellent les débats des féministes à propos des rapports entre les femmes et la technologie. Très sommairement, deux courants s'opposent à propos des technologies en général et de leurs relations aux rapports inéquitables, en termes de genre, dans une société dite patriarcale (van Zoonen, 1992). D'un côté, les «écoféministes » justifient l'opposition des femmes à la technologie en inscrivant ce rejet dans un refus plus global de tout ce qui émane d'une société structurée par la domination d'un genre. Leur argumentation repose sur l'analyse d'une nature différente entre hommes et femmes, et sur l'étrangeté que représente pour ces dernières une technologie qui s'est développée dans un univers guidé par la domination masculine. 
A l'autre extrémité du mouvement féministe, celles que l'on peut, trop rapidement, apparenter au mouvement des «féministes libérales», insistent sur le fait que hommes et femmes pensent similairement et que les femmes doivent rompre avec les schémas qui désignent la technique comme une sphère de compétence exclusivement masculine. Elles devraient dans cette perspective s'adapter à la technique et accéder aux sphères de ce pouvoir sur la matière, pouvoir qui confère aussi un pouvoir socio-économique.

En ce qui concerne les TIC, cette position face à la technologie se traduit, comme pour les zapatistes, par la volonté de mettre en lien sur la «toile» différentes revendications féministes et d'ainsi allier les énergies et de les réunir pour faire triompher plus d'équité. Ce choix manifeste, comme dans le cas précédent, que les femmes ne sont pas intrinsèquement incapables de dominer une technologie construite par les hommes et qu'elles peuvent même la détourner à leur profit. Il s'agit là d'un usage des réseaux qui utilise un canal particulier de communication et de transmission de l'information et en tire parti dans une perspective politique, se rappelant que le réseau est toujours synonyme non seulement de liberté, mais aussi de pouvoir. Même «l'électricité, en courant le long des câbles qui tissent leur toile non seulement entre les points du territoire mais aussi entre les hommes, véhicule bien du pouvoir; un pouvoir multiforme qui prend appui sur le puissance acquise et sur les choses pour restructurer les rapports entre les hommes » (Tinland, 1990: 138).

Ce rapport d'usage à la technologie se double cependant parfois d'un discours qui prône la possibilité d'échapper par la communication virtuelle à un monde réel, perçu comme profondément inique puisqu'il laisse bien peu de place aux identités féminines pour se construire en dehors d'un rapport de domination (voir à ce propos les réflexions menées sur l'éducation différentielle des filles et des garçons). Exister virtuellement apparaît à certaines franges du mouvement féministe comme une solution pour valoriser les capacités des femmes et leur donner l'espace nécessaire en dehors de schémas discriminants. Ainsi, les constats sur l'avantage que la mixité éducative confère principalement aux garçons pourraient-ils être dépassés dans cette perspective par l'étude dans des campus virtuels; les différences entre sexes telles qu'elles sont inscrites dans des corps seraient gommées et chacun serait jugé selon son travail. La virtualisation offrirait dans cette perspective un espace où l'appartenance biologique à un sexe n'enfermerait pas dans une situation de domination, ne serait pas le lieu matériel sur lequel le genre s'inscrirait (Sundén, 2001). Le corps effacé profiterait aux femmes qui pourraient reconstruire ce qu'elles veulent être à travers des identités de cyborgs voyageant, étudiant et travaillant dans un cyberespace.

Dans cette optique, cyborgs asexués ou multisexués, hommes ou femmes correspondraient sur le «net» en tant que monades isolées. Dans ces espaces virtuels, rien ne les distinguerait, rien ne les inscrirait et, de ce fait, rien non plus ne questionnerait les relations que le genre impose. Tout se passe comme si pour rétablir l'équité, il fallait s'abstraire totalement d'un réel inéquitable et ne pouvoir faire l'expérience de la libération d'une société vue comme patriarcale que par l'oubli de son inscription dans le corps et le territoire. Cette expérience de la libération par la virtualisation nous paraît paradoxalement agir comme une réduction de la problématique sociale de la domination d'un genre sur l'autre, et ceci parce qu'elle ne peut être vécue que sur le mode individuel et sur celui de la dénégation. 
En refusant de confronter la question des genres dans le réel, c'est la question de la domination qui a été escamotée.

Il semble bien ainsi qu'en articulant la revendication égalitaire sur la virtualisation des relations, ces perspectives postmodernes nous posent très directement les questions du pouvoir que confèrent ou confisquent les réseaux et celles qui touchent aux modes d'articulation entre monde virtuel et monde réel.

Nier que ce détour par la virtualisation puisse être conçu comme une solution transitoire et individuelle pour se réapproprier, en tant que sujet, une identité fondée sur une expérience de l'équité serait ainsi absurde, mais la portée de ces expériences doit être questionnée. Comment en effet collectiviser dans le monde réel des expériences individuelles de libération par le recours au virtuel? Comment aussi ouvrir à toutes le recours à un tel canal de réappropriation sans mettre en question préalablement les limites que les dispositions construites dans le monde réel ont imposées à l'identité féminine? Ne faut-il pas finalement pour jouer avec les codes, pour détourner en pleine conscience des habitudes et des références, jouir de dispositions qui appartiennent surtout aux groupes qui se trouvent dans la situation des dominants?

Ces interrogations sont loin d'être épuisées dans les débats qui se nouent autour du cyberespace, des «she-cyborgs» et de l'utilisation des réseaux pour porter des revendications identitaires. Elles montrent cependant que ce qui se joue ici est, au-delà de la communication transparente et lisse, une vision de l'humain et du lien social. Valorisant ce qui est dit - le contenu du message - et le canal par lequel une parole transite - les réseaux qui permettent de s'abstraire d'une enveloppe corporelle -, ce cyberféminisme accrédite les visions utopiques et iréniques qui voient dans la désappartenance tant territoriale que sexuelle une issue aux conflits ou tensions entre les groupes et les sexes. Il accrédite ainsi une vision de l'humain qui serait avant tout de l'information enfermée dans une matière vécue comme limitative. Libéré de ce carcan, cet humain hypercérébralisé pourrait alors communiquer en toute transparence et selon ses propres desseins avec un alter ego.

Cet exemple pourrait nous permettre de conclure qu'à travers les métaphores de «réseaux » et de «société de la communication »s'est surtout jouée la question de l'information. Alors si le désenchantement guette peut-être les croyants d'Internet, «la société de l'information» ne semble pas pour autant être une métaphore vide et en déclin. Elle dit peut-être aujourd'hui plus nettement sa vérité qui est la transformation de tout et de l'humain en premier (de son esprit par la réduction de sa connaissance en des savoirs procéduraux, de son corps par l'évidence de l'hybridation) en un code qui peut et doit être productif (cf. Cerqui).

$\mathrm{Ne}$ faudrait-il pas prendre en compte le fait que, pour une bonne part, les sciences de la communication tendent à réduire la société de l'information à une société de communication et à l'analyser exclusivement sous l'angle d'Internet. Or, d'une part, ce dernier ne constitue qu'un aspect de la société de l'information et, d'autre part, cette dernière ne se réduit aucunement à l'aspect communicationnel de l'échange d'informations. Doit-on imputer cela à une méconnaissance des sciences humaines et sociales de la part des experts en TIC? Ou est-ce parce que le débat sur les NTIC est d'ores et déjà stabilisé en un discours dominant consensuel, dans le cadre duquel seules des questions relatives aux effets secondaires et non au fond peuvent être posées? 


\section{LE CODE UNIVERSEL...}

A en croire Kemp (1991: 11), «la formation et l'expertise techniques n'entretiennent souvent aucun rapport avec les sciences humaines. Le technicien est formé pour résoudre des problèmes précis d'ordre scientifique et technique, dans un langage spécialisé qui n'est apte qu'à formuler de tels problèmes et leurs solutions. Le langage technique est incapable de poser ou de résoudre les questions éthiques qui apparaissent quand on prend en compte les hommes qui doivent vivre avec les technologies. Il peut seulement dire comment l'homme, dans des conditions données, agira de la manière la plus efficace». D'où la nécessité de développer en parallèle au savoir technique «un savoir pratique sur la manière de bien vivre avec autrui et pour autrui» (1991:11). A l'heure où nombre de technologies telles que génie génétique, clonage ou encore procréation assistée agissent au cœur même du vivant, ce type de savoir se développe en effet. Force est toutefois de constater que ce savoir est limité au seul champ des biotechnologies. Malgré le fait que les TIC soient toujours décrites comme devant entraîner une révolution, les controverses sont extrêmement peu présentes dans ce domaine.

Dans le projet «Adaptive Brain Interfaces» $(\mathrm{ABI})$, coordonné par la Commission Européenne, les chercheurs ont mis au point une interface cerveau-ordinateur basée sur l'analyse de l'électroencéphalogramme par le biais d'électrodes fixées à un bonnet. A en croire sa brochure de présentation, le projet a entre autres pour but de permettre aux personnes handicapées d'accéder aux services offerts par la société de l'information. Plus généralement, selon l'un des responsables du projet, le but est d' «améliorer l'interface entre l'humain et la machine, pour mieux communiquer avec cette dernière, mais aussi avec d'autres personnes à distance ». De telles assertions préfigurent un projet de société qui demande un humain flexible, malléable et mouvant (cf. Berthoud).

De plus, prônant la transparence, la libre appartenance et l'échange sans ancrage, les métaphores réticulaires et communicationnelles ont contribué à construire une image de l'humain qui est avant tout un code numérique, une somme de parties qui sont toutes réductibles à des informations que l'on peut s'approprier, partager, échanger et vendre. Et si nous devions constater que ces métaphores de la communication horizontale et de l'avenir radieux par la communication ne sont plus suffisantes pour porter le récit et la fiction technique, il n'en demeurerait pas moins qu'en contribuant à déréaliser le réel, elles ont assis au centre de nos grands récits l'information.

A notre époque il est communément admis que la science peut tout expliquer «en décomposant la complexité du monde en éléments simples, puis en reconstruisant de la complexité. Mais une complexité que le scientifique maîtrise cette fois, puisqu'il en est le grand organisateur » (Scardigli, 2001: 137-138), l'individu est lui-même divisible en informations: "Les individus sont devenus des dividuels et les masses, des échantillons, des données, des marchés ou des banques» (Deleuze, 1990: 112). Un tel processus de déconstruction conduit inéluctablement à une indifférenciation du sujet (cf. Besnier), tout comme celle du savoir conduit à une perte de sens.

Institut d' anthropologie et de sociologie

Université de Lausanne 


\section{RÉFÉRENCES}

Bakis, Henry (1993). Les réseaux et leurs enjeux sociaux. Paris: PUF (Que sais-je?).

Bell, Daniel (1976). Vers la société postindustrielle. Paris: Laffont (première publication en anglais en 1973).

- (1999). «The Axial Age of Technology. Foreword: 1999», in The Coming of Post-Industrial Society. A Venture in Social Forecasting. New York: Basic Books, pp. ix-lxxxv.

Berthoud, Gérald, Cerqui, Daniela, Clément, Fabrice, Ischy, Frédéric et Simioni, Olivier (2000). La "société de l'information»: une idée confuse?. Université de Lausanne: Institut d'anthropologie et de sociologie, «Pratiques et théories des sciences et des techniques » no VI.

Berthoud, Gérald, Ischy, Frédéric et Simioni, Olivier (2002). La société de l'information : la nouvelle frontière?. Rapport final adressé au Fond national suisse de la recherche scientifique (subvention no 12-55445.98).

Boltanski, Luc et Eve Chiapello (1999). Le nouvel esprit du capitalisme. Paris: Gallimard.

Bressand, Albert et Catherine Distler (1995). La planète relationnelle. Paris: Flammarion.

Carey, James W. et John J.Quirk (1989). «The History of the Future», in Carey, J. (ed), Communication as Culture. Essays on Media and Society. Boston: Unwin Hyman, pp. 173-200 (première publication en 1973).

Deleuze, Gilles (1990). «Les sociétés de contrôle». L'Autre Journal: 111-114.

Duff, Alistair S. (2000). Information Society Studies. Londres, New York: Routledge.

Fassa, Farinaz (2002). «Apprendre ...pour être fragile», in Travail et fragilisation mentale. Lausanne: Payot.

Flichy, Patrice (2001a). L'imaginaire d'Internet. Paris: La Découverte.

- (2001b). «La place de l'imaginaire dans l'action technique. Le cas de l'Internet». Réseaux 109: 51-73.

Gouldner, Alvin (1989). «Pour une sociologie réflexive». La revue du MAUSS 4: 11-25.

Goody, Jack (1979). La raison graphique. La domestication de la pensée sauvage. Paris: Minuit (première publication en anglais en 1977).

- (1986). La logique de l'écriture. Aux origines des sociétés humaines. Paris: Colin (première publication en anglais en 1986).

Kemp, Peter (1997). L'irremplaçable. Paris: Cerf.

Lyotard, François (1979). La condition postmoderne. Rapport sur le savoir. Paris: Minuit.

Marvin, Carolyn (1988). When Old Technologies Were New. Oxford: Oxford University Press.

Mattelart, Armand (2000). «L'âge de l'information: genèse d'une appellation non contrôlée». Réseaux 101: 21-52.

Mattelart, Armand (2001). Histoire de la société de l'information. Paris: La Découverte.

Miquel, Christian et Ménard, Guy (1988). Les ruses de la technique : le symbolisme des techniques à travers l' histoire. Paris: Meridiens Klincksieck, Montréal: Les Editions du Boréal.

Musso, Pierre (1998). «La philosophie des réseaux», in Le discours philosophique. Paris: PUF, pp. 2321- 2337.

Parrocchia, Daniel (1993). Philosophie des réseaux, Paris: PUF.

Ricœur, Paul (1997). L'idéologie et l' utopie. Paris: Seuil.

Robbins, Kevin et Franck Webster (1999). Times of the Technoculture. From the Information Society to the Virtual Life. London: Routledge.

Scardigli, Victor (2001). Un anthropologue chez les automates. De l'avion informatisé à la société numérisée. Paris: PUF.

Shannon, Claude E. (1949). The Mathematical Theory of Communication. Urbana: The University of Illinois Press. 
Simioni, Olivier (2000). «La société de l'information: émergence et multiplication des discours », in Berthoud G., Cerqui, D., Clément, F., Ischy, F. et Simioni, O., La «société de l'information»: une idée confuse?. Université de Lausanne: Institut d'anthropologie et de sociologie, «Pratiques et théories des sciences et des techniques » no VI, pp. 45-76.

Sundén, Jenny (2001). «What happened to Difference in Cyberspace? The (Re)turn of the SheCyborg». Feminist Media Studies 1: 215-232.

Tinland, Franck (1990). «Réseau et pouvoir : l'électricité relie, aussi, les hommes », in Breton, P. et alii (éds), La technoscience en action. Eléments pour une archéologie du XXe siècle. Seyssel: Champ Vallon, pp. 130-139.

Union européenne (1997). Construire la société européenne de l'information pour tous. Rapport final du groupe d'experts de haut niveau, Commission européenne, Emploi et affaires sociales, CEV/8-97-001-FR-C.

Van Zoonen, Liesbet (1992). «Feminist theory and information Technology». Media, Culture and Society 14: 11-29.

Wiener, Norbert (1952). Cybernétique et société. Paris: Deux Rives. 